\title{
Association between Vestibular Function and Hearing Outcome in Idiopathic Sudden Sensorineural Hearing Loss
}

\author{
Ho-Seok Lee, Ji-Nam Song, Jung Mee Park, Kyoung Ho Park, Hyun bum Kim, and Jae-Hyun Seo \\ Department of Otolaryngology-Head and Neck Surgery, College of Medicine, The Catholic University of Korea, Seoul, Korea
}

$\begin{array}{ll}\text { Received } & \text { November 11, } 2014 \\ \text { Revised } & \text { December 4, } 2014 \\ \text { Accepted } & \text { December 5, } 2014\end{array}$

Address for correspondence

Jae-Hyun Seo, MD

Department of Otolaryngology-

Head and Neck Surgery,

Bucheon St. Mary's Hospital,

College of Medicine,

The Catholic University of Korea,

327 Sosa-ro, Wonmi-gu,

Bucheon 420-717, Korea

Tel +82-32-340-7093

Fax +82-32-340-2674

E-mail revivalseo@catholic.ac.kr
Background and Objectives: To investigate whether different vestibular function tests such as cervical vestibular evoked myogenic potential (cVEMP) and caloric test were correlated with severity, pattern and prognosis in idiopathic sudden sensorineural hearing loss (ISSNHL). Subjects and Methods: Ninety-two patients with unilateral ISSNHL were subjected to complete audiovestibular evaluation. cVEMP and caloric test results were compared with patients' initial and final audiogram. We classified patients in 4 groups as cochlear nerve (C) type, cochlear and superior vestibular nerve $(\mathrm{C}+\mathrm{S})$ type, cochlear and inferior vestibular nerve $(\mathrm{C}+\mathrm{I})$ type and cochlear, superior vestibular nerve, inferior vestibular nerve $(\mathrm{C}+\mathrm{S}+\mathrm{I})$ type, for evaluation of the results. cVEMP and caloric tests were compared among the groups. Results: Abnormal caloric test results and abnormal cVEMP results were found in $50 \%$ and $31.6 \%$ patients, respectively. Multivariate analysis showed that abnormal caloric result (canal paresis) is a significant negative prognostic factor. Conclusions: Initial vestibular function test can be valuable in predicting the final outcome in patients with ISSNHL.

Korean J Audiol 2014;18(3):131-136

\section{Introduction}

Idiopathic sudden sensorineural hearing loss (ISSNHL) is characterized by a sensorineural hearing loss of greater than $30 \mathrm{~dB}$ in more than 3 contiguous audiometric frequencies, that occurs within 3 days. ${ }^{1)}$

One in 10000 to 15000 is afflicted with ISSNHL and nearly half of the population complains of simultaneous vertigo. ${ }^{1,2)}$ Vertigo is reportedly a well known independent predictable value of ISSNHL. ${ }^{3,4)}$

Vestibular involvement in ISSNHL is hypothesized as the extension of the disease due to its anatomical proximity and the correlation of the cochlea and vestibulae. ${ }^{5)}$ Vestibular function test results tend to be abnormal in ISSNHL. Several clinical studies were performed to evaluate the vestibular function in patients with ISSNHL. Some reports indicated that hypoactive caloric response in electronystagmography (ENG) in ISSNHL patients may be of negative prognostic value. ${ }^{1,6)}$ However, it

This is an Open Access article distributed under the terms of the Creative Commons Attribution Non-Commercial License (http://creativecommons. org/licenses/by-nc/3.0/) which permits unrestricted non-commercial use, distribution, and reproduction in any medium, provided the original work is properly cited. is still not clear whether an abnormal caloric test result is predictive of ISSNHL. ${ }^{7-9)}$

Whereas ENG may be used for evaluating superior vestibular integrity and lateral semicircular canal function, cervical vestibular evoked myogenic potential (cVEMP) is a method for investigating inferior vestibular nerve and saccular functional integrity. ${ }^{10)}$ Vestibular evoked myogenic potential (VEMP) is an established test to evaluate a number of central or peripheral vestibular disorders, ${ }^{11)}$ hence recent studies have included the cVEMP test in the evaluation of patients with ISSNHL. Chen and Young ${ }^{12)}$ found 21\% ISSNHL patients with cVEMP abnormality. Iwasaki, et al. ${ }^{13)}$ found that cVEMP abnormality occurred in 77\% ISSNHL patients with vertigo. Stamatiou, et al. ${ }^{11)}$ showed $30.2 \%$ of cVEMP abnormalities in their study. Park, et al. ${ }^{6}$ and Wilson, et al. ${ }^{1)}$ found that ISSNHL patients with canal paresis had a poorer prognosis.

We wished to understand the relationship between the results of vestibular function test (cVEMP and ENG) and the severity, pattern of the initial hearing loss and prognosis of the hearing outcome in ISSNHL patients. 


\section{Subjects and Methods}

\section{Study subjects}

We reviewed the medical records of 92 ISSNHL patients treated in the Department of Otorhinolaryngology-Head and Neck Surgery, Bucheon St. Mary's Hospital, South Korea between December of 2010 and February of 2014. All patients were diagnosed as idiopathic unilateral sensorineural hearing loss of $30 \mathrm{~dB}$ or more, over 3 contiguous audiometric frequencies and had cVEMP and ENG during hospitalization. All other pathologies that were evident on magnetic resonance imaging and any structural abnormalities, including chronic otitis media or inner ear abnormalities, were excluded. Steroid treatment comprised of high dose methyl-prednisone P.O. $(0.8 \mathrm{mg} /$ $\mathrm{kg} /$ day) for 5 days and tapered for 5 days; while other medications administered included peripheral vasodilator and Ginko biloba extracts. For patients with unfavorable initial steroid therapy results who desired to receive another treatment, additional intra-tympanic steroid (dexamethasone) was injected 4 times in 2 weeks. This study was approved by the Institutional Review Board of Bucheon St. Mary’s Hospital.

\section{Audiometry and hearing outcome}

Repeated pure tone audiometry was carried out every other day for each admitted patient. Pure tone averages (PTA) were calculated by averaging the pure tone levels at $500 \mathrm{~Hz}, 1000$ $\mathrm{Hz}, 2000 \mathrm{~Hz}$, and $3000 \mathrm{~Hz}$. Hearing loss was classified in 5 degrees by air conduction: mild (PTA $25-40 \mathrm{~dB}$ ), moderate (PTA 41-55 dB), moderately severe (PTA 56-70 dB), severe (PTA 71-90 dB), and profound (PTA $>90 \mathrm{~dB}$ ). Pretreatment audiogram configurations were categorized into 1 of 5 types according to the classification of Martini, et al. ${ }^{14)}$ The following types of audiograms were defined: 1) Low frequency ascending type, is defined as an audiogram where the difference between the poorer low frequency thresholds and better high frequency ones is $>15 \mathrm{~dB}$. 2) Mid frequency U-shaped type, audiogram where the difference between the poorest thresholds in the mid-frequencies and those at higher and lower frequencies is $>15 \mathrm{~dB}$. 3) High frequency descending, in contrast to the low frequency, higher frequency $>15 \mathrm{~dB}$ worse from the lower frequency. 4) Flat type, the difference between the mean of $250 / 500 \mathrm{~Hz}$ threshold, the mean of $1 / 2 \mathrm{kHz}$ thresholds and the mean of $4 / 8 \mathrm{kHz}$ thresholds is $<15 \mathrm{~dB}$. 5) Total deafness, hearing loss of $\geq 100 \mathrm{~dB}$ at $0.5,1,2$, and $4 \mathrm{kHz}$. Two months after initial treatment, patients went through follow up pure tone audiometry, and the hearing outcome was assessed according to Siegel's criteria; 1) Complete recovery for final hearing better than $25 \mathrm{~dB}$ regardless of the initial hearing. 2) Partial recovery for $>15 \mathrm{~dB}$ of gain or final hearing level between 25 and $45 \mathrm{~dB}$. 3) Slight improvement for $>15 \mathrm{~dB}$ of gain and final hearing worse than $45 \mathrm{~dB}$. 4) No improvement was classified as $<15 \mathrm{~dB}$ of gain and final hearing worse than $75 \mathrm{~dB} \cdot{ }^{15)}$

\section{Vestibular function test}

All patients went through bi-thermal caloric and cVEMP tests to evaluate their vestibular function. Bi-thermal caloric test based on delivering a temperature change to the horizontal semicircular canal, evaluates lateral semicircular afferents. While the cVEMPs, stimulated with clicks on the sternocleidomastoid muscle stimulates and activates the saccular afferents;

Bi-thermal caloric test, was performed with an Aqua stim water caloric irrigator (Micromedical Technology, Chatham, IL, USA). Water applied on the subjects was warm at $44^{\circ} \mathrm{C}$ and cold at $30^{\circ} \mathrm{C}$. The maximum slow phase velocity of nystagmus induced by caloric stimulation of the right or left side was calculated and recorded. Percent of canal paresis (CP) was calculated by the Jonkee's formula $[(\mathrm{RW}+\mathrm{RC})-(\mathrm{LW}+\mathrm{LC}) / \mathrm{RW}+$ $\mathrm{RC}+\mathrm{LW}+\mathrm{LC} \times 100]$. We regarded a $\mathrm{CP}>20 \%$ as abnormal.

VEMP testing was undertaken using a Biologic Navigator PRO (Biologic System Corp., Mundelein, IL, USA). The subjects were asked to sit in an upright position with their heads maintained contra-laterally from the stimulated ear to achieve adequate and constant contraction of the sternocleidomastoid muscle; they were required to maintain muscular efforts in the recording periods ${ }^{16)}$ Two active electrodes were located symmetrically over the midpoint of each sternocleidomastoid muscle. Reference electrodes were placed on the forehead and a ground electrode was applied to the middle of the forehead. Ipsilateral sternocleidomastoid muscle responses to monaural stimuli were recorded. Acoustic stimuli were delivered through headphones. The first positive wave form deflection was marked as $\mathrm{P} 1$ and the first negative deflection was marked as N1. The latencies and amplitudes of these waveforms (P1$\mathrm{N} 1)$ were measured in each subject. The side to side differences in VEMP amplitude of $>20 \%$ or absence in either side of amplitude was regarded as an abnormal VEMP symmetry.

\section{Statistical analysis}

We used SPSS 17.0 (SPSS software, SPSS Inc., Chicago, IL, USA) for statistical methods. A one way between subject analysis of variance (ANOVA) was used for characteristics among the groups and the types of vestibular lesions. The likelihood ratio test and the method of Bonferroni were used to determine statistical significance. For each test, a $p$ value of $\leq 0.05$ was treated as statistically significant. 


\section{Results}

Clinical characteristics of the patients were presented in (Table 1). Ninety-two patients with documented ISSNHL were enrolled in the study, including 55 men and 37 women. Vertigo was present in 52 and absent in 40 patients. By Siegel's criteria of hearing outcomes, ${ }^{15)}$ complete recovery was shown in 35 patients, partial recovery in 19 , slight recovery in 10 , and no improvement in 28. In cVEMP and ENG testing, the incidence of abnormal VEMP response was 29 of 92 and that of abnormal canal paresis was 46 of 92 .

There were no significant differences among groups in respect to the severity of initial hearing loss ( $p$ value $=0.620$ ).
There were no differences in groups according to pure tone types, gender ( $p$ value $=0.711)$, vertigo ( $p$ value $=0.828)$, and the side affected ( $p$ value $=0.808)$ (Table 1).

We classified ISSNHL patients with normal cVEMP results and a normal caloric response as the cochlear nerve (C) type; those with normal cVEMP results but abnormal caloric responses as the cochlear and superior vestibular nerve $(\mathrm{C}+\mathrm{S})$ type; those with abnormal cVEMP results but normal caloric responses as the cochlear and inferior vestibular nerve $(\mathrm{C}+\mathrm{I})$ type and those with abnormal caloric responses and abnormal cVEMP results as the cochlear, superior and inferior vestibular nerve $(\mathrm{C}+\mathrm{S}+\mathrm{I})$ type. Of the 92 patients, $35(38.0 \%)$ were classified as the $\mathrm{C}$ type; 28 (30.4\%) were $\mathrm{C}+\mathrm{S}$ type; $11(11.9 \%)$

Table 1. Patients' demographics $(n=92)$

\begin{tabular}{|c|c|c|c|}
\hline & \multirow{2}{*}{$\mathrm{n}(\%)$} & \multicolumn{2}{|c|}{ Significance } \\
\hline & & Chi-square & $p$ value \\
\hline Gender & & 1.378 & 0.711 \\
\hline Male & $55(59.8)$ & & \\
\hline Female & $37(40.2)$ & & \\
\hline Age range (years) & $51.21 \pm 16.37$ & 3.25 & 0.517 \\
\hline Affected ear & & 0.963 & 0.808 \\
\hline Right & $36(39.1)$ & & \\
\hline Left & $56(60.9)$ & & \\
\hline Vertigo & & 0.890 & 0.828 \\
\hline Presence & $52(56.6)$ & & \\
\hline Absence & $40(43.5)$ & & \\
\hline Hearing loss & & 2.109 & 0.620 \\
\hline Mild & $9(9.78)$ & & \\
\hline Moderate & $21(22.83)$ & & \\
\hline Moderately severe & $19(20.65)$ & & \\
\hline Severe & $26(28.26)$ & & \\
\hline Profound & $17(18.48)$ & & \\
\hline Initial pure tone audiogram type* & & 5.328 & 0.902 \\
\hline Low frequency ascending & $26(28.2)$ & & \\
\hline Mid frequency $U$ shaped & $6(6.52)$ & & \\
\hline High frequency descending & $21(22.82)$ & & \\
\hline Flat & $27(29.34)$ & & \\
\hline Total deafness & $12(13.04)$ & & \\
\hline
\end{tabular}

Table 2. Comparison of initial hearing level based on the result of vestibular assessment

\begin{tabular}{|c|c|c|c|c|c|c|}
\hline & \multicolumn{6}{|c|}{ No. according to degrees of initial hearing loss } \\
\hline & Mild & Moderate & Moderately severe & Severe & Profound & Total \\
\hline C & 3 & 8 & 8 & 11 & 5 & 35 \\
\hline$C+S$ & 4 & 7 & 4 & 7 & 6 & 28 \\
\hline $\mathrm{C}+\mathrm{I}$ & 1 & 1 & 2 & 6 & 1 & 11 \\
\hline $\mathrm{C}+\mathrm{S}+\mathrm{I}$ & 1 & 5 & 5 & 2 & 5 & 18 \\
\hline Total & 9 & 21 & 19 & 26 & 17 & 92 \\
\hline
\end{tabular}

The chi-square test did not present statistical significance ( $p$ value=0.917). $C$ : cochlear nerve type, l: inferior vestibular nerve type, S: superior vestibular nerve type 
were $\mathrm{C}+\mathrm{I}$ type and 18 (19.57\%) were $\mathrm{C}+\mathrm{S}+\mathrm{I}$ type.

We tested for a correlation among the groups and the severity of initial hearing loss. No statistically significant relationship was found among them ( $p$ value $=0.917)$ (Table 2, Fig. 1). Then, we compared the groups and pure tone audiometry type of initial hearing loss. There were also no statistically significant correlation $(p$ value $=0.944)($ Table 3$)$.

The mean values of initial PTA on the affected sides were $67.54 \mathrm{~dB}$ in type $\mathrm{C}, 69.13 \mathrm{~dB}$ in type $\mathrm{C}+\mathrm{S}, 76.14 \mathrm{~dB}$ in type $\mathrm{C}+\mathrm{I}$, and $72.63 \mathrm{~dB}$ in type $\mathrm{C}+\mathrm{S}+\mathrm{I}$. No statistically significant

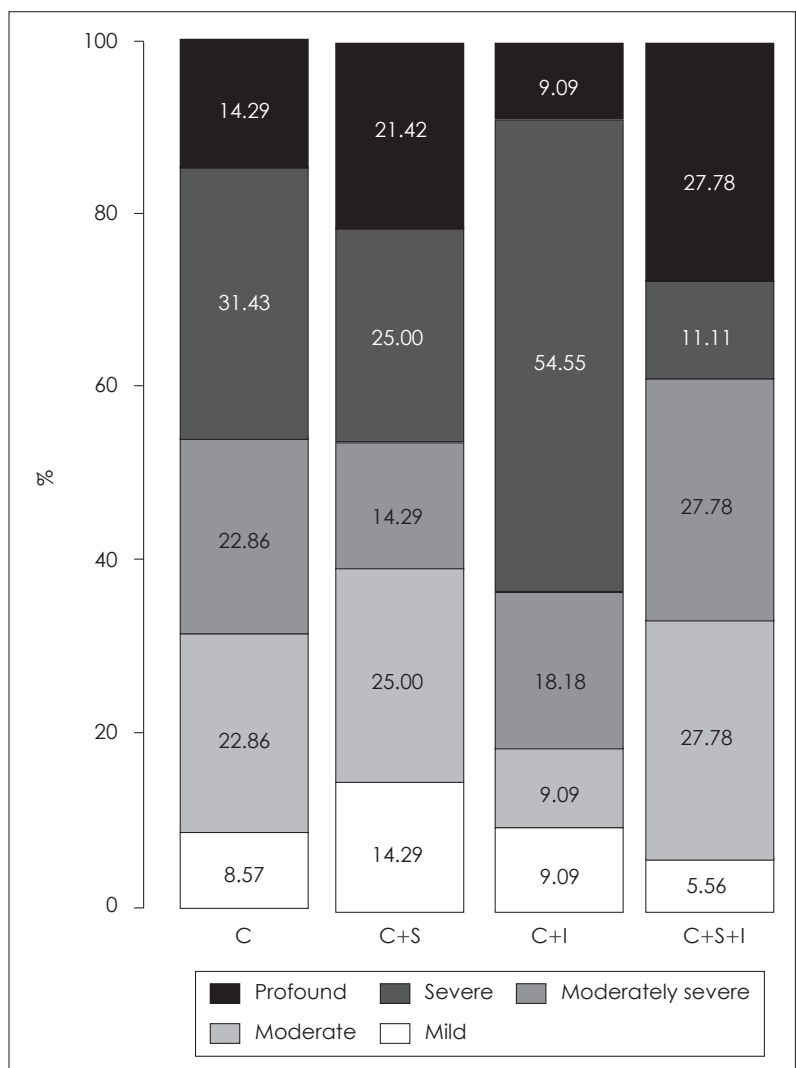

Fig. 1. Comparison of initial hearing level based on the results of vestibular assessment. The hearing levels among the groups are well distributed. The chi-square test did not present statistical significance ( $p$ value $=0.917)$. C: cochlear nerve type, l: inferior vestibular nerve type, $\mathrm{S}$ : superior vestibular nerve type. correlation was noted among them ( $p$ value $=0.730$ ). Two months after initial treatment, the final PTAs were $43.54 \mathrm{~dB}$ in type C, $50.04 \mathrm{~dB}$ in type $\mathrm{C}+\mathrm{S}, 39.32 \mathrm{~dB}$ in type $\mathrm{C}+\mathrm{I}$, and $60.00 \mathrm{~dB}$ in type $\mathrm{C}+\mathrm{S}+\mathrm{I}$. There were no statistically significant differences in pure tone findings ( $p$ value $=0.265$ ). However, there was a statistically significant difference between initial mean PTA and final PTA when we analyze by ANOVA ( $p$ value=0.029). We accordingly assessed these results as Bonferroni correction method. The $\mathrm{C}+\mathrm{I}$ group had better hearing outcomes than the $\mathrm{C}+\mathrm{I}+\mathrm{S}$ group $(23.84 \mathrm{~dB}, p$ value $=0.021)$. There were no other significant differences among groups. The hearing outcomes of each group were also compared by Siegel's criteria. ${ }^{15)}$ There was no statistically significant result $(p$ value $=0.917)($ Fig. 2, 3).

\section{Discussion}

ISSNHL with balance impairment was first reported in 1949. ${ }^{17)}$ However, the pathogenesis, clinical features, and the optimal treatment are still not clear. Over several decades, a

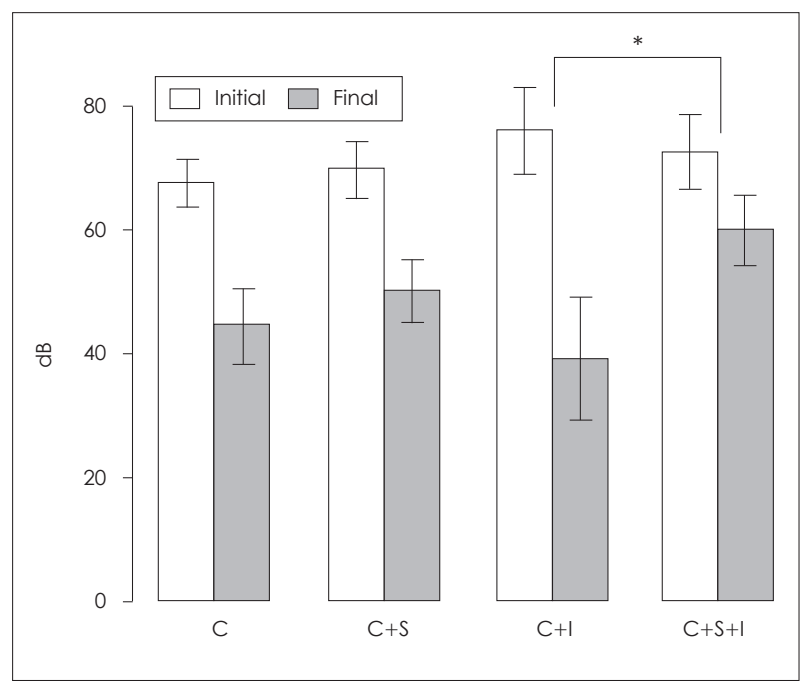

Fig. 2. Mean initial and final pure tone average among the groups. The statistical significance between group $\mathrm{C}+\mathrm{I}$ and group $\mathrm{C}+\mathrm{S}+\mathrm{I}$ is marked with asterisk (Bonferroni adjusted $p$ value $=0.021$ ). $\mathrm{C}$ : cochlear nerve type, I: inferior vestibular nerve type, S: superior vestibular nerve type.

Table 3. Comparisons in the pure tone audiometry type of initial hearing loss based on the results of vestibular function test

\begin{tabular}{|c|c|c|c|c|c|c|}
\hline & \multicolumn{6}{|c|}{ No. according to the types of hearing loss* } \\
\hline & Type 1 & Type 2 & Type 3 & Type 4 & Type 5 & Total \\
\hline $\mathrm{C}$ & 9 & 2 & 8 & 12 & 4 & 35 \\
\hline $\mathrm{C}+\mathrm{S}$ & 10 & 1 & 5 & 8 & 4 & 28 \\
\hline $\mathrm{C}+\mathrm{I}$ & 2 & 2 & 3 & 3 & 1 & 11 \\
\hline $\mathrm{C}+\mathrm{S}+\mathrm{I}$ & 5 & 1 & 5 & 4 & 3 & 18 \\
\hline Total & 26 & 6 & 21 & 27 & 12 & 92 \\
\hline
\end{tabular}

The chi-square test did not present statistical significance ( $p$ value=0.944). *pure tone audiometry type of Martini, et al. ${ }^{14)}$ Type 1: low frequency ascending, Type 2: mid frequency U shaped, Type 3: high frequency descending, Type 4: flat, Type 5: total deafness. C: cochlear nerve type, I: inferior vestibular nerve type, S: superior vestibular nerve type 


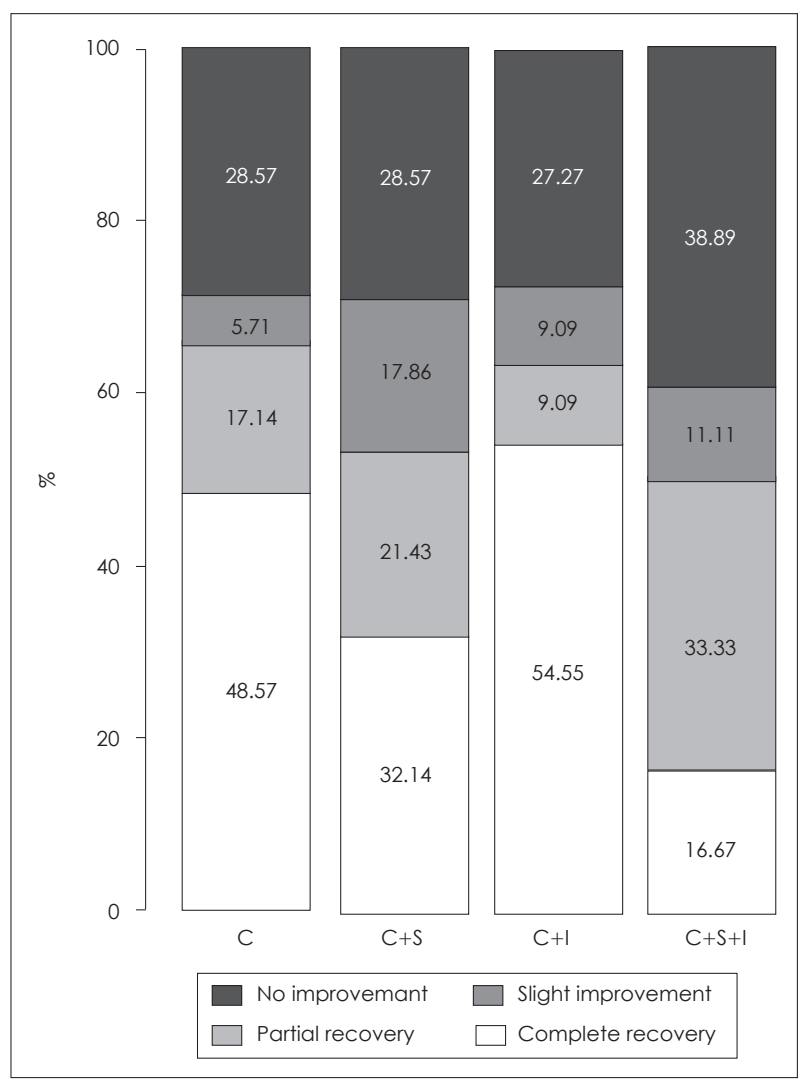

Fig. 3. The distribution of the hearing outcome categorized by Siegel's criteria ${ }^{15)}$ among the groups. The chi-square test did not present statistical significance. C: cochlear nerve type, I: inferior vestibular nerve type, S: superior vestibular nerve type.

number of studies have attempted to elucidate the role of vestibular function in cochlear function impairment, but the study results are still controversial. ${ }^{5)}$

It has been reported that $30-40 \%$ of ISSNHL patients have accompanying vertigo, which is considered as a poor prognostic factor. ${ }^{6}$ Some studies reported that vertigo is more often found in profound hearing loss. ${ }^{3)}$ Due to the anatomical correlation, it can be hypothesized that the profound hearing loss tends to have more damaged vestibular function. We evaluated the vestibular function in ISSNHL with cVEMP and caloric test. cVEMP test is used to determine saccular function, otolithic organ, and the inferior vestibular nerve and central connection. On the other hand, caloric test stimulates the lateral semicircular canal with subsequent evaluation of the superior division of the vestibular nerve. ${ }^{16)}$

We found similar results on reviewing the literature. Park, et al. ${ }^{6)}$ proposed that $\mathrm{CP}$ represents as a poor prognostic result in patients suffering sudden hearing loss with vertigo. Meanwhile, Wang, et al. ${ }^{18)}$ proposed that profound hearing loss with normal VEMP is associated with favorable hearing results. Stamatiou, et al. ${ }^{11)}$ showed that the group with otolithic organ involvement tends to have vertigo more than semicir- cular canal involvement. Khetarpal's ${ }^{19)}$ histopathological study indicated that vestibular organ damage was not correlated with vertigo in ISSNHL patients.

Among the 92 patients diagnosed as ISSNHL in our study, $31.52 \%$ of patients showed abnormal VEMP and $50 \%$ of patients showed abnormal caloric response. These results revealed that the lesion involves the labyrinth in a considerable number of ISSNHL cases.

Of the 4 study groups mentioned, the largest was group $\mathrm{C}$ $(38.0 \%)$, i.e., patients with a lesion confined to a cochlear nerve. The next was group $\mathrm{C}+\mathrm{S}(30.4 \%)$, i.e., patients whose lesion involved the superior vestibular nerve (lateral semicircular canal). $\mathrm{C}+\mathrm{S}+\mathrm{I}$ group (19.57\%) followed, i.e., lesion involving both superior and inferior vestibular nerve (lateral semicircular canal and otolithic organ). The smallest group was $\mathrm{C}+\mathrm{I}$ (11.9\%), i.e., lesion involving inferior vestibular nerve (otolithic organ). These results showed that the superior vestibular nerve and cochlear nerve involvement in ISSNHL was more frequent than those of the inferior vestibular nerve and cochlear nerve $(p$ value $=0.02$ ). There was no statistically significant relationship between labyrinthine involvement and the severity of hearing impairment in both initial PTA and the final PTA ( $p$ value $=0.730$ and $p$ value $=0.265$ for each) .

Multivariate analyses for the comparison of difference between initial and final PTA had statistically significant results among groups ( $p$ value $=0.029$ ). Lesions involving the inferior vestibular nerve and cochlear nerve tended to have favorable prognostic results than those including the inferior vestibular nerve, superior vestibular nerve, and cochlear nerve ( $p$ value $=0.021$ ) (Fig. 2). This result suggested that the more extensive the lesion, especially involving the semicircular canal, the poorer the prognosis. Siegel's classification ${ }^{15)}$ as the assessment tool for comparisons of outcome had no statistically significant results ( $p$ value $=0.391)$. However, vestibular nerve involvement may not have a critical role in the severity of initial hearing loss. This study also showed that the vestibular test abnormality and subjective vertigo were not closely related ( $p$ value $=0.828)$.

\section{Conclusion}

The extent of the disease in ISSNHL does not affect either the severity of initial hearing loss or the type of PTA, but it could influence the hearing outcome. The wider the disease extent in ISSNHL patients, especially with both cVEMP and caloric test area abnormalities, the poorer the hearing outcome. Superior vestibular nerve involvement in the ISSNHL patient is more frequent and produces a worse hearing outcome, as compared to that of the inferior vestibular nerve. But vestibular 
function test as a prognostic tool for ISSNHL is still controversial.

\section{REFERENCES}

1) Wilson WR, Laird N, Kavesh DA. Electronystagmographic findings in idiopathic sudden hearing loss. Am J Otolaryngol 1982;3:279-85.

2) Stokroos RJ, Albers FW. The etiology of idiopathic sudden sensorineural hearing loss. A review of the literature. Acta Otorhinolaryngol Belg 1996;50:69-76.

3) Ito $S$, Fuse $T$, Yokota $M$, Watanabe $T$, Inamura $K$, Gon $S$, et al. Prognosis is predicted by early hearing improvement in patients with idiopathic sudden sensorineural hearing loss. Clin Otolaryngol Allied Sci 2002;27:501-4.

4) Byl FM Jr. Sudden hearing loss: eight years' experience and suggested prognostic table. Laryngoscope 1984;94(5 Pt 1):647-61.

5) Korres S, Stamatiou GA, Gkoritsa E, Riga M, Xenelis J. Prognosis of patients with idiopathic sudden hearing loss: role of vestibular assessment. J Laryngol Otol 2011;125:251-7.

6) Park HM, Jung SW, Rhee CK. Vestibular diagnosis as prognostic indicator in sudden hearing loss with vertigo. Acta Otolaryngol Suppl 2001;545:80-3.

7) Laurikainen E, Aantaa E, Kallinen J. Electronystagmographic findings and recovery of cochlear and vestibular function in patients suffering from sudden deafness with a special reference to the effect of anticoagulation. Audiology 1989;28:262-7.

8) Shaia FT, Sheehy JL. Sudden sensori-neural hearing impairment: a report of 1,220 cases. Laryngoscope 1976;86:389-98.

9) Mattox DE, Simmons FB. Natural history of sudden sensorineural hearing loss. Ann Otol Rhinol Laryngol 1977;86(4 Pt 1):463-80.

10) Colebatch JG, Halmagyi GM, Skuse NF. Myogenic potentials generated by a click-evoked vestibulocollic reflex. J Neurol Neurosurg Psychiatry 1994;57:190-7.

11) Stamatiou G, Gkoritsa E, Xenellis J, Riga M, Korres S. Semicircular canal versus otolithic involvement in idiopathic sudden hearing loss. J Laryngol Otol 2009;123:1325-30.

12) Chen CN, Young YH. Differentiating the cause of acute sensorineural hearing loss between Ménière's disease and sudden deafness. Acta Otolaryngol 2006;126:25-31.

13) Iwasaki S, Takai Y, Ozeki H, Ito K, Karino S, Murofushi T. Extent of lesions in idiopathic sudden hearing loss with vertigo: study using click and galvanic vestibular evoked myogenic potentials. Arch Otolaryngol Head Neck Surg 2005;131:857-62.

14) Martini A, Milani M, Rosignoli M, Mazzoli M, Prosser S. Audiometric patterns of genetic non-syndromal sensorineural hearing loss. Audiology 1997;36:228-36.

15) Siegel LG. The treatment of idiopathic sudden sensorineural hearing loss. Otolaryngol Clin North Am 1975;8:467-73.

16) Vanspauwen R, Wuyts FL, Van De Heyning PH. Validity of a new feedback method for the VEMP test. Acta Otolaryngol 2006;126:796800.

17) Rasmussen H. Sudden deafness. Acta Otolaryngol 1949;37:65-70.

18) Wang CT, Huang TW, Kuo SW, Cheng PW. Correlation between audiovestibular function tests and hearing outcomes in severe to profound sudden sensorineural hearing loss. Ear Hear 2009;30:110-4.

19) Khetarpal U. Investigations into the cause of vertigo in sudden sensorineural hearing loss. Otolaryngol Head Neck Surg 1991;105:36071. 